\title{
SPRAY DROPLET CHARACTERIZATION USING A PIEZOELECTRIC SENSOR THROUGH CLASSIFICATION BASED ON MACHINE LEARNING
}

\author{
I

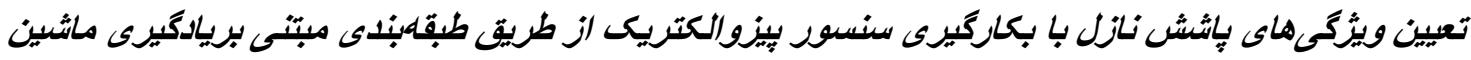 \\ Hassan Poorvousooghi Gargari ${ }^{1}$, Rahman Farrokhi Teimourlou ${ }^{\star 1)}$, Morteza Valizadeh ${ }^{2)} 1$ \\ 1) Urmia University, Department of Mechanical Engineering of Biosystems, Iran \\ 2) Urmia University, Department of Electronics and Computer Engineering, Iran \\ Tel: 00989141398929; E-mail: r.farrokhi@urmia.ac.ir \\ DOI: https://doi.org/10.35633/inmateh-59-17
}

Keywords: Classification, Deposit, Machine Learning, Piezoelectric, Spray Droplet

\begin{abstract}
Nowadays, the indiscriminate use of pesticides for plant protection, has led to severe environmental pollution. This input accounts for a portion of the agricultural economy and should be sprayed in a way that has the highest biological efficacy and the least run-off. Therefore, real-time evaluation of spray characteristics and its classification is necessary. In the current research, a piezoelectric sensor was employed for detection of vibration signals from impaction of droplets to the active surface of the sensor. To supervised classification, the Support Vector Machine classifier as a Machine Learning model was implemented by means of extracted features from conditioned signals. By using a feature selection algorithm, six features were selected comprising mean, median, mode of signal peaks, root mean square, mean deviation and impulse factor of the signals. These features used as Support Vector Machine inputs. Model targets were spray droplet characteristics that were determined using image processing techniques on water sensitive papers. The results showed that the Linear and medium Gaussian models have the highest overall accuracy. Linear Support Vector Machine has higher accuracy and precision for training data (94.60\% and $94.63 \%$ ) and its model was able to predict with $92.59 \%$ accuracy. Precision of classifier model was higher than $92 \%$ for all classes. The highest miss rate of the model was approximately $15 \%$ in the separation of class $C$. Accurate and precise performance of linear classifier was confirmed by determining the Kappa coefficient of 0.77 .
\end{abstract}

\begin{tabular}{|c|c|}
\hline & 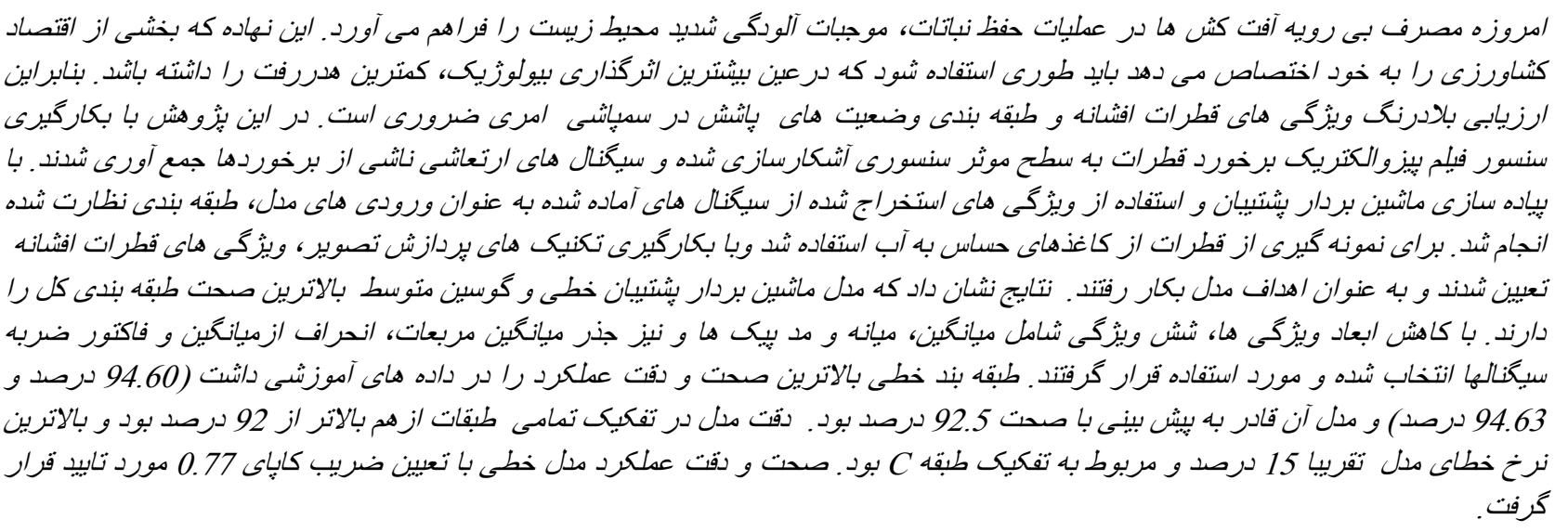 \\
\hline
\end{tabular}

\section{INTRODUCTION}

Despite the disadvantages of using pesticides, while development and research on various methods of plant protection is ongoing, spraying is still the most effective and fastest method. Unfortunately, pesticide application techniques are such that even in the most optimistic way, too much of the pesticides enter the environment, disrupts natural balance and causes pests and diseases to emerge.

\footnotetext{
${ }^{1}$ Hassan Poorvousooghi Gargari, Ph.D. Stud.; Rahman Farrokhi Teimourlou, As Prof.; Morteza Valizadeh, As Prof.
} 
In addition, it is not getting the desired result, its environmental and health destructive effects appear over time. About 30 to 50 percent of the sprayed solution is dissipated by drift and pouring on the soil (Fallahjeddi, 2005). Also, it is difficult to determine how droplets deposits on the plant surfaces have the least run off and the highest biological efficacy. Limiting the use of chemical pesticides to the extent possible in the context of Integrated Pest Management programs and their impact on the actual goals is the basis of optimal pest management (Mathews, 2008). Indiscriminate use of pesticides and their detrimental environmental impacts of spraying on various targets (e.g. pests, diseases or weeds) and the excessive waste of pesticides as an economically expensive input doubles the importance of spraying monitoring.

Methods to determine spray characteristics are typically low-precise, time-consuming and sometimes costly. The use of water sensitive papers (WSPs) is a common and practical method for estimating spray characteristics with relatively acceptable results with the advent of digital imaging and processing techniques. Expensive laser instruments and high-speed imaging in spray studies is a relatively new and precise method. The subject of atomization, spraying, and impaction of droplets to the surface have complex natures and is influenced by many parameters. Therefore, by conducting theoretical studies and using computational fluid dynamics (CFD) and experimental tests, some mathematical and numerical models are presented as well.

The time-consuming and cost-effective tests have led many researchers to find suitable alternatives such as the development of computer simulations as their main advantage is their repeatability. Spray quality is a term used to describe the properties of sprayed droplets, which can be assessed by estimation of parameters such as droplet size, spray density and deposition rate. So, the fine droplets tend to be most prone to drift and the coarse droplets, due to their high kinetic energy, penetrate better through the foliage, but are more likely to run off from the plant surfaces (Minov et. al., 2016). Koch and Knewitz (2008) studied methods for determining the spray deposition and distribution in the vineyards. They concluded that although artificial samplers such as WSPs don't provide a detailed image of the droplet deposition, are more common due to the ease of handling and use. Zhu et al (2011) launched a portable scanning system for evaluating of spray deposit distribution which includes an image processing software, laptop computer and handheld card scanner. The scanning resolution can be increased up to $2400 \mathrm{dpi}$, which enables it to detect minimum 17 micron droplets. Canopy-spray interaction models were formed for predicting impaction behaviour at the leaf surface by scanning plant leaves and creating virtual leaf surface models and virtual spray models. The results revealed that the droplet retention increased with decreasing surface tension, static contact angle, droplet size and its velocity (Dorr et. al., 2014). Delele et. al. (2016) investigated the dynamic impact behaviour of spray droplets on plant surfaces by using a high-speed camera imaging technique with multiphase computational fluid dynamics model and volume of fluid (VOF) approach. It was reported that the process is highly influenced by the surface and droplet characteristics during impact. The various combinations of Weber, Reynolds and Ohnesorge numbers determine the droplet maximum spread factor and the number of secondary droplets produced in the impaction. These criteria can be used on a farm scale to determine droplet deposition and drift models to better understand agricultural spraying operations. A sensor was developed for measuring low volume spray deposit. The sensor surfaces had parallel conductive lines that varied the voltage of the sensor according to the droplet deposition on the surface. Two types of sensor surface with constant and variable line spacing were tested. Results showed that R-squared for the constant width line sensor and variable width sensor was 0.901 and 0.934 respectively (Maze and Parekh, 1993). Crow et. al. (2008) developed a digital device for sensing distribution of spray deposition. The system used sensor surfaces with 15 lead conductive elements. The presence of droplets on the sensor surface caused electrical connection and their size and position were detected by the comparator circuit. They concluded by testing that the system was able to detect coarse droplets by using a low reference voltage and stated that the technique needed further investigation. A spray deposition sensing system was developed and evaluated preliminary. The sensor surface consisted of several parallel tin-plated copper traces of variable gap widths. The investigations showed that there was a significant difference in the output voltage of the sensor with different deposits and droplets size. Multiple sensing configurations and further experiments were needed to separate out the effects of application rate versus droplet sizes (Kesterson et. al., 2015). A system based on solution conductivity theory was developed to quickly measure the accumulating droplet mass of sprays. The relationship between accumulating droplet mass and solution conductivity was found as a linear regression relationship with high correlation and coefficient of determination of 0.99 . The relative error of this system compared with WSPs was $7.75 \%$ and droplet collection and recovery rates were $84 \%$ 
and 91\%, respectively (Su et. al., 2017). Wang et. al. (2019) monitored pesticide droplet deposition with a novel capacitance sensor. The results indicated that there was a linear relationship between the deposition mass due to the impact of droplets on the sensor surface and the output voltage of the sensor. $R$-squared values for the ionized and non-ionized solutions were 0.835 and 0.882 , respectively.

Piezoelectric sensors have piezoelectric materials as a sensor element. They can create electricity when subjected to a mechanical stress. They will also work in reverse, generating a strain by the application of an electric field. The piezoelectric effect can be defined by the Eq.1.

Where:

$$
D=d * T+\varepsilon * E_{c}
$$

$D$ is the electric charge displacement, $d$ is the piezoelectric coefficient, $T$ is the applied stress, $\varepsilon$ is the electric permittivity and $E_{c}$ is the electric field strength. PVDF (Polyvinylidene fluoride) film sensors have high voltage output ten times higher than piezo ceramics for the same input force. They also have the advantages of being thin and flexible and have wide frequency response, and good linearity.

WSPs are impregnated with bromophenol blue solution, which as a result of the impact of the droplets, stains are recorded on them. The papers are mounted on plant targets or on special stands. After spraying and drying, they are collected and digital images are prepared. Then, they are analyzed using image processing techniques. Volume Median Diameter (VMD) refers to the midpoint droplet size, where half of the spray volume is in droplets smaller, and half of the volume is in droplets larger than the mean. The spray deposition can be estimated by computing the volume of all droplets per unit area (Matthews, 2008).

Support Vector Machine (SVM) first introduced by Vapnik and Chervonenkis in 1971, is a machine learning algorithm based on statistical learning theory and is a kind of structural risk minimization based algorithms and a supervised learning model. It is optimized overall and is used today as a robust and reliable classification tool. The nature of the SVM could be of two types, either linearly separated data with the largest distance, or by using nonlinear kernel functions map data into a high dimensional space to seek a separate hyperplane. Then it can perform classification by using the constructed N-dimensional hyperplane that optimally separates data (Vapnik, 1998).

In this study, by using the approach of droplet impaction to surfaces and detecting the minor forces originated by impactions, vibration signals were acquired, filtered and processed. By extracting the features from the signals and implementing machine learning techniques, the classification of the sprays was performed in terms of the spray characteristics. The main objective is to provide a method or tool to evaluate the spraying characteristics for different nozzle status.

\section{MATERIALS AND METHODS}

The main part of electronic setup is comprised of a piezoelectric polymer (PVDF) sensor model LDT1028K manufactured by Measurement Specialties Inc. which formed a cantilever beam by using of two jaw holders (Fig. 1). The sensor specifications are listed in Table1.

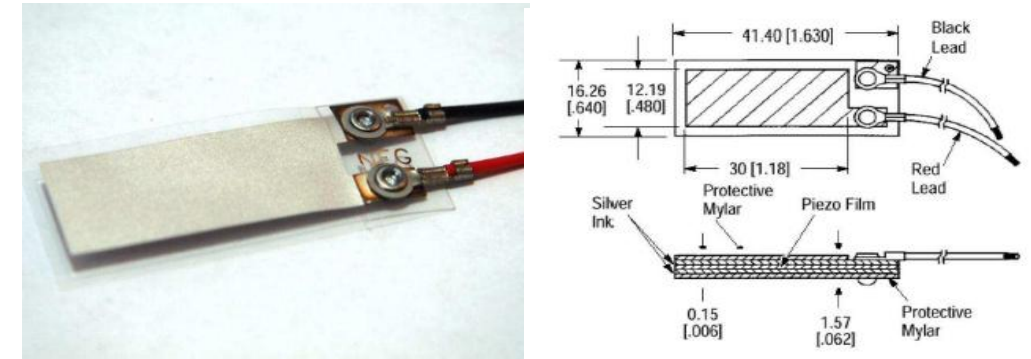

Fig. 1 - LDT01-28K film sensor and its dimensions in $\mathrm{mm}$ [inches]

Table 1

LDT1-028K PVDF film sensor specifications

\begin{tabular}{lc}
\hline Minimum Impedance & $1 \mathrm{M} \Omega$ \\
Preferred Impedance & $10 \mathrm{M} \Omega$ and higher \\
Output Voltage & $10 \mathrm{mV}-100 \mathrm{~V}$ depending on force and circuit impedance \\
Storage Temperature & $-40^{\circ} \mathrm{C}-+70^{\circ} \mathrm{C}\left[-40^{\circ} \mathrm{F}-60^{\circ} \mathrm{F}\right]$ \\
Operating Temperature & $0^{\circ} \mathrm{C}-+70^{\circ} \mathrm{C}\left[32^{\circ} \mathrm{F}-60^{\circ} \mathrm{F}\right]$ \\
\hline
\end{tabular}


To increase the local resolution of the sensor, a sliding plastic plate with $10 \times 10 \mathrm{~mm}^{2}$ aperture was installed on sensor so that $1 \mathrm{~cm}^{2}$ effective sensor surface is created in order to decrease the number of spray droplets impact or avoid multiple impacts. It relatively ensures that each sensor signal will be produced from one droplet. An ARDUINO board was used as an analog to digital converter and microcontroller. Signal amplifier module was designed using Proteus Pro 8.8 software and after it was built, it was mounted on the ARDUINO board (Fig.2).
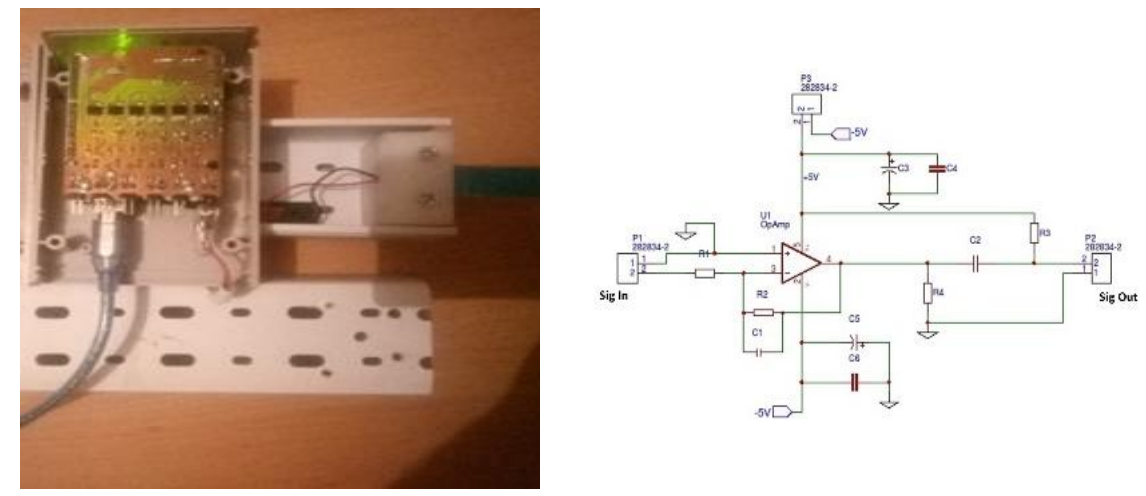

Fig. 2 - Amplifying module on board and its circuit

LM358A operational amplifiers were used to amplify the signals. The data acquisition system was developed using the ARDUINO and serial communication script on the board side, as well as the Excel Macro on the laptop computer side, which could handle sampling frequency about $600 \mathrm{~Hz}$. To reduce the noise effects on the signals, a shielded cable was used to transmit the signal, and a USB shielded cable was used to connect the board to the laptop computer.

Despite the development of new atomization and spraying techniques such as centrifugal and airassisted techniques, hydraulic nozzles are used in most countries for spraying. The size and distribution of the droplets in these nozzles is very non-uniform and the application rate is very high. Hollow cone nozzles are classified into three types of spiral, axial whirl and tangential whirl, and the first type is mostly used in the agricultural applications (Fig.3).

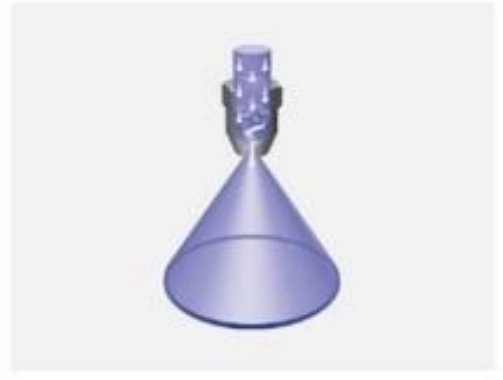

Axial Whirl

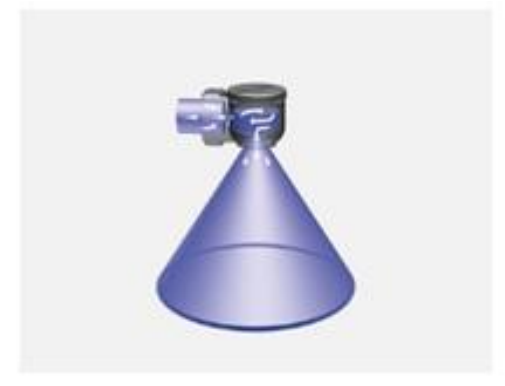

Tangential Whirl

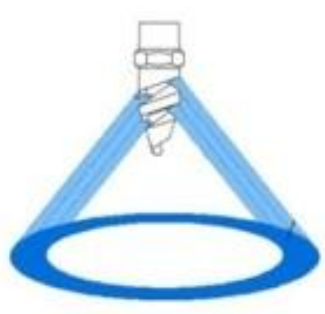

Spiral

Fig. 3 - Three types of hollow cone nozzles

To perform the spraying tests, a laboratory spraying setup was provided including a tank, centrifugal pump, barometer, and adjustable lance equipped with a hollow cone nozzle. By twisting Lance handle, five spraying status were determined including I, II, III, IV and V. The tap water was used as the spray liquid and performed primary tests using WSPs as droplet sampling surfaces and sensor system as a means of sensing vibration signals. To control the spraying time length, a metal base frame was provided with a sliding plastic plate as a barrier. The frame was located closer to the nozzle and in front of it. For sampling with WSPs and Sensor, barrier plate was raised and released. All spraying tests were carried out in the same spraying condition. The samples of vibration signals due to droplets impaction are shown in Fig. 4. 


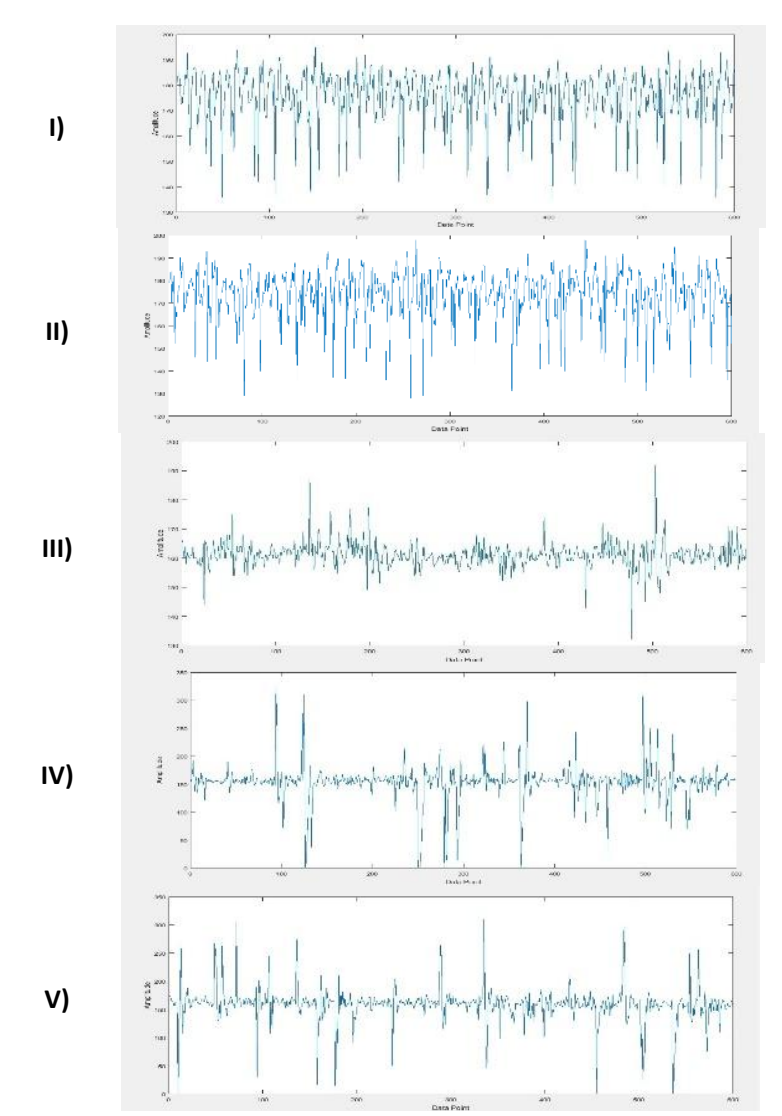

Fig. 4 - Samples of vibration signals for five spraying status

Spray droplets were sampled by WSP with 30 replications for each spray status. The papers were scanned and digital images were obtained (Fig.5). By using image processing algorithm developed by coding in MATLAB environment it was able to identify objects and their properties by performing low-level and midlevel processing with segmentation and labelling functions (Poorvousooghi Gargari, 2013). By analyzing the papers, the five spray classes were separated in terms of VMD and spray deposition. These classes were used as SVM targets.

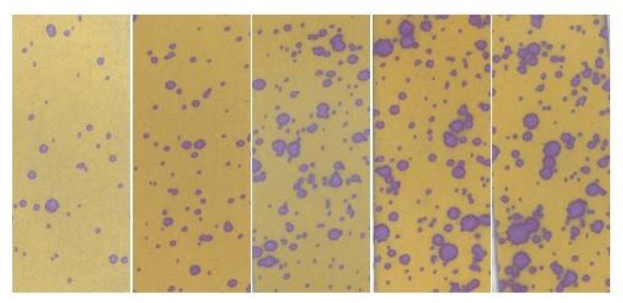

Fig. 5 - Digital images of water sensitive papers

Signal processing includes signal conditioning and extraction of features from the signals. Initially, by applying the moving average filter and the band-stop filter, the signals were smoothed and filtered. Ninety signals with one second duration were selected for each spraying status. Twenty six features were extracted using a developed algorithm that includes peak detection functions, statistics and vibration features computing functions. By means of peak analysis, these features were extracted:

Mean of Peak values is the central value of a discrete set of values (Eq.2).

$$
\mu_{p}=\frac{\sum_{m=1}^{M} p_{m}}{M}
$$

Median of Peak values is the value separating the higher half from the lower half of samples (Eq.3).

$$
\operatorname{median}_{p}=l+\frac{h}{f}\left(\frac{M}{2}-c\right)
$$


Where:

$l$ is lower class boundary of median class, $h$ is size of median class, $f$ is frequency corresponding to the median class, $M$ is sum of frequencies and $c$ is cumulative frequency preceding median class.

Mode of peak values is the value that appears most often (Eq.4).

$$
\operatorname{mode}_{p}=l+h\left(\frac{f_{m}-f_{1}}{2 f_{m}-f_{1}-f_{2}}\right)
$$

Where:

$l$ is lower class boundary of modal class, $h$ is size of modal class, $f_{m}$ is frequency corresponding to the modal class, $f_{1}$ is frequency proceeding the modal class and $f_{2}$ is frequency proceeding the modal class.

Some important statistical and vibration features extracted from the signals and their equations are listed in Table 2.

Table 2

Some important features extracted from signals

\begin{tabular}{|c|c|c|c|}
\hline Feature & Equation & Feature & Equation \\
\hline Mean & & $\begin{array}{c}\text { Mean } \\
\text { Deviation }\end{array}$ & \\
\hline $\begin{array}{l}\text { Root Mean } \\
\text { Square }\end{array}$ & & $\begin{array}{l}\text { Moment (order } \\
\text { i) }\end{array}$ & \\
\hline Kurtosis & & $\begin{array}{l}\text { Impulse } \\
\text { Factor }\end{array}$ & \\
\hline
\end{tabular}

By means of reducing the volume of SVM inputs, the processing speed can be increased, which reduces the time required for modeling and increases precision. To dimensionality reduction of the features, Correlation-based Feature Subset Evaluation algorithm and Best First method in Weka software were used. More efficient and main features were selected including mean, median, mode of peak values, root mean square, mean deviation and impulse factor of signals. The features matrix was created and $70 \%$ of the data were considered as training data and $30 \%$ as test data. In other words from $90^{*} 6$ features for each spraying Status, $63^{*} 6$ were used as training data and $27^{*} 6$ as test data.

Classification was implemented using the SVM classifier and by cross validation method in MATLAB software. The learning process was performed using SVM classifier with different kernel functions on the training data, the models were tested using test data and two most accurate learning models were extracted. The confusion matrix was used to analyze the classifier performance. In this matrix, the criteria such as Accuracy, Sensitivity, Specificity and Precision were used as the overall evaluation of the classifiers. The equations of these criteria are as follows:

$$
\begin{aligned}
& \text { Accuracy }=\frac{T P+T N}{T P+F N+F P+T N} \\
& \text { Sensitivity }=\frac{T P}{T P+F N} \\
& \text { Specificity }=\frac{T N}{F N+T N} \\
& \text { Precision }=\frac{T P}{T P+F P}
\end{aligned}
$$

Where:

$T P$ is the number of true positive instances, $T N$ is the number of true negative instances, $F P$ is the number of false positive instances and $F N$ is the number of false negative instances.

Cohen's kappa coefficient is used as criterion for further evaluation of classifier performance and indicates the degree of agreement between predicted and actual instances for all classes (Eq.9).

$$
\mathbb{K}=\frac{P_{0}-P_{e}}{1-P_{e}}
$$

Where:

$P_{0}$ is the observed relative agreement among actual values and prediction values and $P_{e}$ is the hypothetical probability of chance agreement or probability that both prediction and actual values are positive or negative. Interpretation of Kappa coefficient is presented in Table 3. 
Kappa interpretation guidelines of Landis and Koch (1977)

Table 3

\section{RESULTS}

\begin{tabular}{cc}
\hline Kappa statistic & Strength of Agreement \\
\hline$<0$ & Poor \\
$0.01-0.20$ & Slight \\
$0.21-0.40$ & Fair \\
$0.41-0.60$ & Moderate \\
$0.61-0.80$ & Substantial \\
$0.81-1.00$ & Almost Perfect
\end{tabular}

The analysis of water sensitive papers indicated that the averaged VMD for the five spraying status were estimated to be $580,621,719,903$ and $1267 \mu \mathrm{m}$. Also, the averaged spray depositions were 0.52 , $0.65,1.26,1.47$ and $4.07 \mu \mathrm{l} / \mathrm{cm}^{2}$. This points out that wisting the lance handle changes the nozzle status and thus increases the droplet size and the spray deposition. It is clear that the increase in droplet size has a slight slope in three first statuses and is evident in status IV and is sharp in status V. In the case of spray deposition, since the volume is theoretically directly related to the third power of the diameter, the slope of the increase is very significant. These results show that five spray classes were separable by using image processing technique including A,B,C,D and E. These classes were used for SVM classifier targets. Table 4 presents spray classes and their specifications.

Table 4

Spray classes and their specifications

\begin{tabular}{cccc}
\hline $\begin{array}{c}\text { Nozzle } \\
\text { status }\end{array}$ & $\begin{array}{c}\text { Averaged } \\
\text { VMD }\end{array}$ & $\begin{array}{c}\text { Averaged } \\
\text { Deposition }\end{array}$ & $\begin{array}{c}\text { Spray } \\
\text { Class }\end{array}$ \\
\hline I & 580 & 0.52 & $\mathrm{~A}$ \\
II & 621 & 0.65 & $\mathrm{~B}$ \\
III & 719 & 1.26 & $\mathrm{C}$ \\
IV & 903 & 1.47 & $\mathrm{D}$ \\
V & 1267 & 4.07 & $\mathrm{E}$ \\
\hline
\end{tabular}

Signal processing, feature extraction and finally feature selection provide data for use as SVM inputs. The averaged features of signals for each nozzle status are presented in Table 5.

Table 5

The averaged features of Signals

\begin{tabular}{ccccccc}
\hline $\begin{array}{c}\text { Nozzle } \\
\text { status }\end{array}$ & $\boldsymbol{\mu}_{\boldsymbol{p}}$ & $\boldsymbol{m e d i a n}_{\boldsymbol{p}}$ & mode $_{\boldsymbol{p}}$ & $\boldsymbol{R} \boldsymbol{M S}$ & $\boldsymbol{M D}$ & $\boldsymbol{I F}$ \\
\hline I & 193.47 & 193.38 & 188.10 & 189.15 & 2.59 & 1.06 \\
II & 192.19 & 192.05 & 186.92 & 187.75 & 2.66 & 1.07 \\
III & 179.77 & 179.10 & 177 & 177.66 & 1.74 & 1.09 \\
IV & 181.14 & 175.64 & 170.50 & 171.88 & 9.61 & 1.60 \\
V & 189.12 & 182.88 & 177.04 & 177.88 & 9.69 & 1.62 \\
\hline
\end{tabular}

The result of SVM classifier implementation shows that the overall accuracy of classifier with Linear, Quadratic, Cubic, Fine Gaussian, Medium Gaussian and Coarse Gaussian kernel functions are 94.6, 91.1, 90.5, 91.7, 94.3 and $89.2 \%$, respectively. Linear and Medium Gaussian have the highest performance accuracy in training and was further investigated. The prediction models were obtained and tested with test data.

The overall evaluation criteria of the SVM classifier with linear and medium Gaussian kernels are presented in Table 6. 
Table 6

The overall evaluation criteria of the SVM classifiers

\begin{tabular}{ccccccc}
\hline & Data & Accuracy & Sensitivity & Specificity & Precision & Kappa \\
\hline \multirow{2}{*}{ Linear kernel } & Training & 94.60 & 94.60 & 98.65 & 94.63 & 0.83 \\
& Test & 92.59 & 92.59 & 98.15 & 92.58 & 0.77 \\
& Training & 94.29 & 94.29 & 98.57 & 94.31 & 0.82 \\
\multirow{2}{*}{ Gaussian kernel } & Test & 90.37 & 90.37 & 97.59 & 90.56 & 0.70 \\
\hline
\end{tabular}

Based on data from table 6, overall 92.58 percent of the cases classified by the linear model are accurate. Overall precision of classifier for predicting is $92.58 \%$. SVM with linear kernel has performed better on training data and the evaluation criteria are great, SVM with Gaussian kernel performed much better in the training process but predicting with the obtained model has been weaker. The values of kappa coefficient are within the range of $0.61-0.80$ that indicate the overall substantial agreement between the predicted and the target instances and validate classifier performance. These values for training show almost perfect agreement.

However, the values of the overall classification criteria are desirable and acceptable. Therefore, the selected features can be used as SVM inputs to reduce the computational load of the processor and reduce the prediction time. The classification error using the linear kernel is up to $7.41 \%$. Table 7 presents the sensitivity and precision of the linear classifier for each spray class.

Table 7

The Sensitivity and Precision of classifier for each class

\begin{tabular}{ccccc}
\hline Class & \multicolumn{2}{c}{ Sensitivity } & \multicolumn{2}{c}{ Precision } \\
\hline & Training Data & Test Data & Training Data & Test Data \\
A & 96.83 & 96.30 & 96.83 & 92.86 \\
B & 93.65 & 92.59 & 95.16 & 92.59 \\
C & 92.06 & 85.19 & 93.55 & 92.00 \\
D & 93.65 & 92.59 & 90.76 & 92.59 \\
E & 96.83 & 96.30 & 96.83 & 92.86 \\
\hline
\end{tabular}

The linear SVM model is less sensitive to class $C$ separation but in separation of class $A$ and $E$ is very sensitive. Miss rate (False Negative Rate) of linear SVM model in separation of class $C$ from other 4 classes is highest (approximately $8 \%$ for training data and $15 \%$ for test data). Precision of classifier model is higher than $92 \%$ for all class separation. Low precision of classifier in separating class $D$ from class $C$ in training is due to its low accuracy in the separation of class C. ROC (Receiver Operating Characteristics) curves were obtained for test data that show that specificity is close to 100 in all classes and minimum sensitivity is $85 \%$ in Class C. Area Under Curve (AUC) for this class is within acceptable and good range (80-90) and classifying model is sufficiently reliable (Fig.6).

Although classification of spray droplet has not been reported so far, spraying quality evaluation has been investigated with often expensive techniques in several papers. Sun et. al. (2017) reported a relative system error less than $7.75 \%$ in a system that works based on solution conductivity compared with WSPs. Baijing and $\mathrm{Li}$ (2011) used spectral technique and regression models to predict droplet concentration with an average error of 6.41. The method proposed by Yang Jun et. al. (2017) using the LiDAR technique for detection of spray droplet distribution had relative error less than $7 \%$ compared with WSPs. 


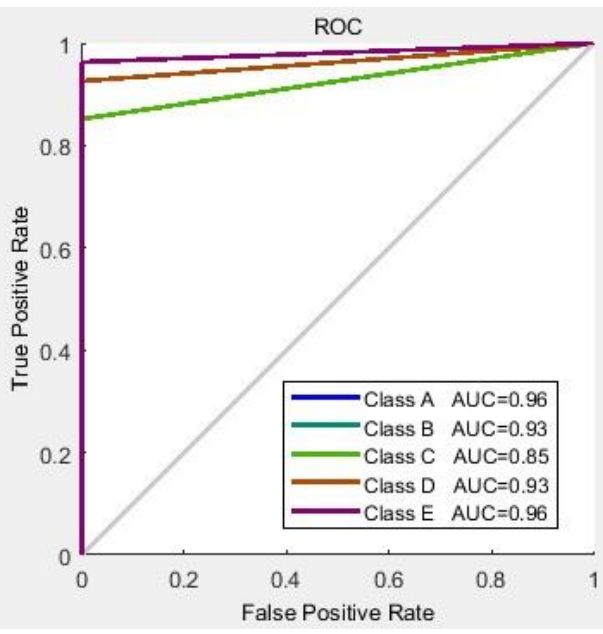

Fig. 6 - ROC curves for all classes in training data

\section{CONCLUSIONS}

The research was conducted for the nozzle spraying characterization in agricultural activities. Analysis of vibration signals as a result of spray droplets impaction to the active sensor surface was considered. Spraying tests were carried out by using an adjustable nozzle. Spray droplets were sampled with water sensitive papers, and specifying characteristics were identified using image processing techniques. Feature extraction and selection algorithms were used for determination of effective signal features. The Support Vector Machine (SVM) classifier was implemented with various kernel functions for supervised classification and their performances were studied. As a consequence, the conclusion is summarized below:

1) Results of machine learning process showed that accuracy and precision of SVM models with various kernel functions were acceptable. Therefore, SVM models have high potential for classification of spraying status.

2) Linear SVM model has higher overall accuracy and precision than others. The model is capable of separating the spray classes with $92.59 \%$ overall accuracy. Kappa coefficient for the model was 0.77 which indicates substantial agreement in classification. Within class analysis, Precision of classifier model is higher than $92 \%$ for all class separation. The lowest sensitivity of model is related to class C. Miss rate for class $\mathrm{C}$ was the highest value (approximately $15 \%$ ).

3) Due to the advantages such as low cost, real time outputs, easy set up and faster execution of the method, it can be uses as efficient and useful tool for the spray characterization in fields and testing centres. Manufacturer of nozzles and spraying equipment can also use it for preparation of nozzle instructions and guidelines.

\section{REFERENCES}

[1] Baijing Q., Lei Y., (2011), Non-contact measurement of droplets concentration on the leaf based on Vis/NIR spectroscopy. Fuzzy Systems and Knowledge Discovery (FSKD), Eighth International Conference;

[2] Crowe T., Downey D., Giles D., (2005), Digital device and technique for sensing distribution of spray deposition. Transactions of the ASAE, vol.48(6), pp.2085-2093;

[3] Delele M. A., Nuyttens D., Duga A., Ambaw A., Lebeau F., Nicolai B., Verboven P., (2016), Predicting the dynamic impact behaviour of spray droplets on flat plant surfaces. Soft Matter, vol.12(34), pp.7195-7211;

[4] Dorr G.J., Kempthorne D.M., Mayo L.C., Forster W.A., Zabkiewicz J.A., McCue S.W., Hanan J., (2014), Towards a model of spray-canopy interactions: interception, shatter, bounce and retention of droplets on horizontal leaves. Ecological Modeling, vol.290, pp.94-101;

[5] FallahJeddi R., (2005), Calibration of conventional sprayers in IRAN. AREEO Pubs. Karaj. Iran, (in Farsi);

[6] Kesterson M.A., Luck J.D., Sama M.P., (2015), Development and Preliminary Evaluation of a Spray Deposition Sensing System for Improving Pesticide Application. Sensors, vol.15(12), pp.31965-31972;

[7] Koch H., Knewitz H., (2014), Methodology and sampling technique of spray deposit and distribution measurement in vineyards. Nachrichtenbl. Deut. Pflanzenschutzd, 60(2); 
[8] Landis J.R.; Koch G.G., (1977), The measurement of observer agreement for categorical data. Biometric, vol.33(1), pp.159-174;

[9] Matthews G., (2008), Pesticide application methods: John Wiley \& Sons.

[10] Maze R. C., Parekh K.T., (1993), Sensor for measuring low volume spray deposit. Paper presented at the American Society of Agricultural Engineers Meeting (USA);

[11] Measurement Specialties Inc., (2013), Piezo Film Sensors. Technical Manual;

[12] Minov S.V., Cointault F., Vangeyte J., Pieters J.G., Nuyttens D., (2016), Spray Droplet Characterization from a Single Nozzle by High Speed Image Analysis Using an In-Focus Droplet Criterion. Sensors, vol.16(2), p.218;

[13] Poorvousooghi Gargari H., (2013), Study the Performance of Electrostatic Spraying System on Leaf Surfaces using Image Processing. MSc Dissertation. Bu-Ali Sina University. IRAN. (in Farsi);

[14] Sun C., Qiu W., Ding W., Gu J., (2017), Design and Experiment of a Real-Time Droplet Accumulating Mass Measurement System. Transactions of the ASABE, vol.60(3), pp.615-624;

[15] Vapnik V., (1998), Statistical Learning Theory: John Wiley and Sons, New York;

[16] Wang P., Yu W., Ou M., Gong C., Jia W., (2019), Monitoring of the Pesticide Droplet Deposition with a Novel Capacitance Sensor. Sensors, vol.19(3), p.537;

[17] Yongjun Z., Shenghui Y., Lan Y., Hoffmann C., Chunjiang Z., Liping C., Yu T., (2017), A novel detection method of spray droplet distribution based on LIDARs. International Journal of Agricultural and Biological Engineering, vol.10(4), pp.54-65;

[18] Zhu H., Salyani M., Fox R.D., (2011), A portable scanning system for evaluation of spray deposit distribution. Computers and Electronics in Agriculture, vol.76(1), pp.38-43. 